\title{
ASPECTOS EPIDEMIOLÓGICOS DA HANSENÍASE EM UM MUNICÍPIO NORDESTINO DO BRASIL
}

\author{
Epidemiological aspects of leprosy in a municipality in northeastern Brazil \\ Aspectos epidemiológicos de la lepra en un municipio del Noreste de Brasil
}

\author{
Carmem Rita Sampaio de Sousa \\ Universidade Federal do Ceará - UFC - Fortaleza (CE) - Brasil
}

Mariana Campos da Rocha Feitosa (iD

Universidade Federal do Ceará - UFC - Fortaleza (CE) - Brasil

\author{
Ana Beatriz Ferreira Pinheiro \\ Secretaria Municipal de Saúde de Maracanaú - Maracanaú (CE) - Brasil
}

Kellyn Kessiene de Sousa Cavalcante

Universidade Federal do Ceará - UFC - Fortaleza (CE) - Brasil

\section{RESUMO}

Objetivo: Analisar os aspectos epidemiológicos da hanseníase em um município nordestino do Brasil. Métodos: Realizou-se um estudo transversal a partir de dados secundários sobre a hanseníase oriundos do Sistema de Informação de Agravos de Notificação (Sinan) do município de Maracanaú, Ceará, de 2009 a 2018. Os dados foram analisados pelo software Stata, versão 11.2. Resultados: Diagnosticaram-se 639 casos novos de hanseníase no município de Maracanaú. A maioria dos casos novos foi do sexo masculino. A classificação clínica da doença predominante é a forma dimorfa, com proporção média de $42,4 \%$. Dos 639 casos diagnosticados em Maracanaú, 33 (5,2\%) ocorreram através de exame de contato, 23 (3,6\%) por exame de coletividade e $583(91,2 \%)$ pelas formas de detecção passiva. Conclusão: O município encontra-se com taxa de detecção anual que indica hiperendemicidade, indicando alta detecção de casos, com registro em menores de quinze anos, indicando a permanência de fontes de transmissibilidade. As características clínicas e epidemiológicas são do sexo masculino, classificação operacional multibacilar, sendo a forma clínica dimorfa a mais frequente e com predomínio para o grau zero de incapacidade no momento do diagnóstico.

Descritores: Hanseníase; Epidemiologia; Monitoramento Epidemiológico.

\begin{abstract}
Objective: To analyze the epidemiological aspects of leprosy in a municipality in northeastern Brazil. Methods: A cross-sectional study was conducted with secondary data on leprosy from the Notifiable Disease Information System (Sistema de Informação de Agravos de Notificação - Sinan) of the municipality of Maracanaú, Ceará, from 2009 to 2018. The data were analyzed using Stata software version 11.2. Results: A total of 639 new cases of leprosy were diagnosed in the municipality of Maracanaú. Most of the new cases were men. The predominant clinical classification of the disease is the dimorphic form, with a mean rate of $42.4 \%$. Of the 639 cases diagnosed in Maracanaú, 33 (5.2\%) were identified by contact examination, 23 (3.6\%) by collective examination and $583(91.2 \%)$ by passive detection. Conclusion: The municipality has an annual detection rate that indicates hyperendicity, thus indicating high case detection found in children under fifteen years old and permanence of transmission sources. The clinical and epidemiological characteristics point to the male sex, multibacillary operational classification, the most common being the dimorphic clinical form, and predominantly zero degree of disability at the time of diagnosis.
\end{abstract}

Descriptors: Leprosy; Epidemiology; Epidemiological Monitoring.

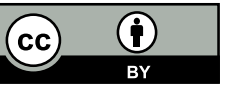




\section{RESUMEN}

Objetivo: Analizar los aspectos epidemiológicos de la lepra en un municipio del noreste de Brasil. Métodos: Se ha realizado un estudio transversal de los datos secundarios de la lepra a través del Sistema de Información de Agravios de Notificación (Sinan) del municipio de Maracanaú, Ceará, entre 2009 y 2018. Se analizaron los datos por el software Stata, versión 11.2. Resultados: Se ha diagnosticado 639 casos nuevos de la lepra en el municipio de Maracanaú. La mayoría de los casos nuevos ha sido del sexo masculino. La clasificación clínica de la enfermedad que ha predominado ha sido la forma dimorfa con la proporción media del 42,4\%. De los 639 casos diagnosticados en Maracanaú, 33 (5,2\%) se dieron por la prueba de contacto, 23 (3,6\%) por la prueba de colectividad y $583(91,2 \%)$ por las formas de detección pasiva. Conclusión: La tasa de detección anual del municipio indica hiperendemicidad con elevada detección de casos con el registro en menores de quince años lo que indica la permanencia de fuentes de transmisibilidad. Las características clínicas y epidemiológicas son del sexo masculino, la clasificación operacional multibacilar y la forma clínica dimorfa es la más frecuente con el predominio para el grado cero de discapacidad en el momento del diagnóstico.

Descriptores: Lepra; Epidemiología; Monitoreo Epidemiológico.

\section{INTRODUÇÃO}

A hanseníase se caracteriza como um processo infeccioso causado pelo bacilo Mycobacterium leprae, que tem como especificidade a capacidade de infectar um grande número de indivíduos, mas apenas um número pequeno desses indivíduos apresenta manifestações clínicas ${ }^{(1)}$.

Diante disso, é um incitante problema de saúde pública por seu impacto socioeconômico e sua repercussão psicológica decorrente do processo do adoecimento ${ }^{(2)}$. Apesar dos esforços do Ministério da Saúde e de instituições internacionais de saúde para sua eliminação mediante estratégias e ações programáticas, a transmissão ativa dessa doença continua presente ${ }^{(2,3)}$.

Em 2016, segundo a Organização Mundial da Saúde (OMS), 143 países reportaram 214.783 casos novos de hanseníase, o que representa uma taxa de detecção de 2,9 casos por 100 mil habitantes. No Brasil, no mesmo ano, foram notificados 25.218 casos novos, perfazendo uma taxa de detecção de 12,2/100 mil hab. Esses parâmetros classificam o país como de alta carga para a doença, sendo o segundo com o maior número de casos novos registrados no mundo(4).

Na região Nordeste, a taxa de detecção de casos novos por 100 mil habitantes foi de 23,42, destacando-se o estado do Maranhão, com a maior taxa de detecção, de 53,91 casos por 100 mil habitantes, e o estado do Rio Grande do Norte, com a menor taxa de detecção, de 7,89 casos por 100 mil habitantes ${ }^{(4)}$.

No Ceará, a taxa de deteç̧ão de casos novos foi de 22,24 casos por 100 mil habitantes nos anos de 2012 a 2016. Segundo a Secretária Estadual de Saúde, o estado do Ceará notificou 8.536 casos novos da doença, sendo o sexo masculino predominante no número de notificações de casos novos da doença entre 2014 e 2018 (4.909), com uma perceptível redução na taxa de detecção em todas as faixas etárias, porém ainda apresenta quantitativo de casos significativo na população de maiores de 60 anos $^{(4,5)}$.

O Programa Nacional de Controle da Hanseníase (PNCH) orienta ações de comunicação em saúde, educação permanente e mobilização social. Em consonância com as políticas vigentes, deve-se promover a participação de diferentes atores sociais no planejamento, execução e avaliação, favorecendo a democratização e a descentralização dessas ações ${ }^{(6,7)}$.

Diante dos desafios para a execução do Plano Nacional de Eliminação da Hanseníase, os municípios deveriam priorizar a capacitação dos profissionais da rede de Atenção Básica à Saúde ${ }^{(3)}$, bem como a descentralização das ações de hanseníase e o envolvimento dos gestores e equipes municipais de saúde ${ }^{(8)}$.

Dessa forma, o presente estudo teve como objetivo analisar os aspectos epidemiológicos da hanseníase em um município nordestino do Brasil.

\section{MÉTODOS}

Realizou-se um estudo transversal de dados secundários a partir das fichas de notificação de hanseníase do Sistema de Informação de Agravos de Notificação (Sinan), do setor de Vigilância Epidemiológica, da Secretaria de Saúde do município de Maracanaú, Ceará, Brasil. As informações foram coletadas em fevereiro de 2019, sendo 
incluídos todos os casos novos de hanseníase confirmados e notificados no período de janeiro de 2009 a dezembro de 2018.

Os dados demográficos relativos à distribuição anual da população foram obtidos a partir do Instituto Brasileiro de Geografia e Estatística (IBGE). O município de Maracanaú está localizado a cerca de $20 \mathrm{~km}$ da capital do estado, tendo como municípios limítrofes Fortaleza, Caucaia, Maranguape e Pacatuba, e conta com uma área de $105 \mathrm{~km}^{2}$. Em 2018, apresentava população estimada em 226.128 habitantes, eminentemente urbana $(99,69 \%)$, com índice de desenvolvimento humano (IDH) de 0,686 e densidade demográfica de $2.134,13 \mathrm{hab} / \mathrm{km}^{2(9)}$.

A cidade de Maracanaú é historicamente marcada pelo cuidado aos portadores de hanseníase. Em dezembro de 1942, no seu território, inaugurou-se a Colônia de São Bento, posteriormente denominada Colônia Antônio Justa, local destinado a abrigar cerca de 500 doentes do "mal de Hansen". Atualmente, o local não mais se destina a tais cuidados. O Programa Nacional de Controle da de Hanseníase foi implantado na referida cidade com o objetivo de estabelecer metas e diretrizes para ampliar ações de prevenção, diagnóstico, tratamento e controle da doença a partir da reorganização do trabalho de atenção à saúde nas Unidades Básicas de Saúde (UBASF), desenvolvendo ações cujos objetivos são a busca de casos novos na fase inicial da doença, avaliação dos contatos dos pacientes em tratamento, além de descentralização dos atendimentos para as UBASF, de modo a encaminhar apenas os casos mais graves para os médicos de referência.

Identificaram-se 656 fichas individuais de notificação no período analisado, consolidadas pelo Sinan da Secretaria Municipal de Saúde de Maracanaú. Destas, excluíram-se os casos com erro de diagnóstico $(n=8)$, duplicidade $(n=4)$ e inconsistência nos dados $(n=5)$, perfazendo um total de 639 casos novos de hanseníase.

Posteriormente, realizou-se refinamento do banco de dados para a identificação e seleção das variáveis de interesse: faixa etária, sexo, forma clínica da doença, classificação operacional, grau de incapacidade no diagnóstico, modo de entrada e modo de detecção.

Organizaram-se os dados, sendo exportados pelos programas Libre Office e TabWin, versão 3.2, e analisados pelo software Stata, versão 11.2 (Stata Corp LP, College Station, TX, USA). O cálculo das proporções e taxas de deteç̧ão basearam-se nos indicadores das Diretrizes para vigilância e eliminação da hanseníase como problema de saúde pública, elaborado pelo Ministério da Saúde.

Consideraram-se, para a avaliação dos indicadores de monitoramento do progresso da eliminação da hanseníase enquanto problema de saúde pública, os seguintes parâmetros da OMS: proporção de casos de hanseníase segundo sexo entre o total de casos novos (casos de hanseníase do sexo feminino dividido pelo total de casos novos, multiplicado por 100); proporção de casos segundo classificação operacional entre o total de casos novos (casos de hanseníase multibacilares dividido pelo total de casos novos, multiplicado por 100); taxa de detecção anual de casos novos de hanseníase por 100 mil habitantes (casos novos residentes em determinado local e diagnosticados no ano da avaliação dividido pela população total residente, no mesmo local e período, multiplicado por 100 mil); taxa de detecção anual de casos novos de hanseníase na população de zero a 14 anos por 100 mil habitantes (casos novos em menores de 15 anos de idade residentes em determinado local e diagnosticados no ano da avaliação dividido pela população de zero a 14 anos de idade, no mesmo local e período, multiplicado por 100 mil).

Os dois indicadores utilizados para avaliar a qualidade dos serviços de hanseníase foram: proporção de cura de hanseníase entre os casos novos diagnosticados nos anos das coortes (casos novos de hanseníase residentes em determinado local, diagnosticados nos anos das coortes e curados até 31/12 do ano da avaliação, dividido pelo total de casos novos de hanseníase residentes no mesmo local e diagnosticados nos anos das coortes, multiplicado por 100) e proporção de casos novos de hanseníase com grau de incapacidade física avaliada no diagnóstico (casos novos de hanseníase com o grau de incapacidade física avaliada no diagnóstico, residentes em determinado local e detectados no ano da avaliação, dividido pelos casos novos de hanseníase, residentes no mesmo local e diagnosticados no ano da avaliação, multiplicado por 100). As coortes são compostas por casos novos paucibacilares (PB), diagnosticados no ano anterior ao da avaliação, e de contatos dos casos novos multibacilares, diagnosticados dois anos antes da avaliação.

Por se tratar de um estudo sobre dados secundários oficiais de domínio público, sem identificação de sujeitos, houve dispensa de apreciação por Comitê de Ética em Pesquisa.

\section{RESULTADOS}

No período de 2009 a 2018, diagnosticaram-se 639 casos novos de hanseníase no município de Maracanaú, o que equivale a uma taxa média de detecção de 29,3 casos novos para cada 100 mil habitantes. A maioria dos 
casos novos era do sexo masculino ( $n=344 ; 53,8 \%$ ), entretanto as notificações do sexo feminino predominaram nos anos de 2013 (56\%) e 2018 (65\%) (Figura I).

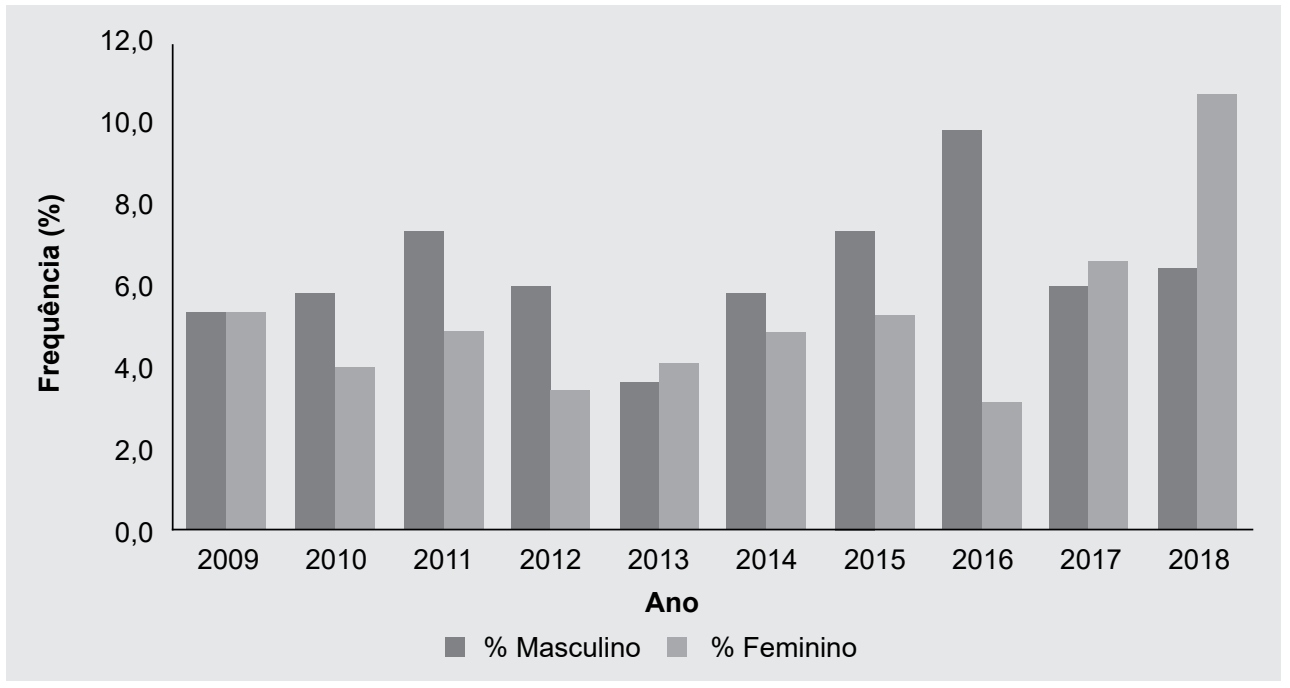

Figura I - Proporção de casos novos de hanseníase por sexo, Maracanaú/CE, 2009 a 2018 (n=639). Maracanaú, 2019.

Fonte: Sinan/ Vigilância Epidemiológica - Secretaria de Saúde de Maracanaú/CE. Dados coletados em 6 de fevereiro de 2019

Quanto à avaliação do grau de incapacidade, avaliou-se mais de $90 \%$ dos casos novos quanto ao grau de incapacidade física no diagnóstico. Destes, $13,6 \%$ tinham grau II, e $60 \%$ dos casos novos passaram por avaliação na alta. Salienta-se que $15,6 \%$ dos casos novos ainda se encontram em registro ativo, sendo $17 \%$ com tratamento iniciado no ano de 2017 e 83\%, em 2018 (Tabela I).

No período de 2009 a 2018, examinaram-se $77 \%$ dos contatos dos casos novos de hanseníase diagnosticados no município de Maracanaú e, nos três primeiros anos avaliados, a proporção de contatos foi acima de 80 , excedendo $90 \%$ em todos os anos posteriores (Tabela I).

A Tabela I demonstra que a classificação clínica da doença predominante é a forma dimorfa, que se classifica como multibacilar, com proporção média de $42,4 \%$. Adicionalmente, verificou-se o modo de entrada dos pacientes predominante, sendo o de casos novos $(86,1 \%)$, entretanto, embora o Ministério da Saúde não especifique parâmetro para avaliação do indicador de recidiva, a proporção foi de $3,1 \%$.

Dos 639 casos diagnosticados em Maracanaú, 33 (5,2\%) ocorreram através de exame de contato, 23 (3,6\%) por exame de coletividade e 583 (91,2\%) pelas formas de detecção passiva (encaminhamento, demanda espontânea e outros modos) (Tabela I).

Tabela I - Distribuição dos casos novos de hanseníase segundo sexo, forma clínica, classificação operacional na notificação, grau de incapacidade no diagnóstico, modo de entrada e modo de deteç̧ão no município de Maracanaú, nos anos de 2009 a 2018. Maracanaú, 2019.

\begin{tabular}{lcc}
\hline Variáveis & $\mathbf{n}$ & $\%$ \\
\hline Sexo & & \\
$\quad$ Masculino & 344 & 53,8 \\
$\quad$ Feminino & 295 & 46,2 \\
Forma clínica na notificação & & 14,6 \\
$\quad$ Indeterminada & 93 & 21,3 \\
$\quad$ Tuberculoide & 136 & 42,4 \\
$\quad$ Dimorfa & 271 & 18,9 \\
Virchowiana & 171 & 1,9 \\
$\quad$ Ignorado/branco & 12 & 0,9 \\
Não classificada & 6 & 34,7 \\
Classificação operacional na notificação & & 65,3 \\
$\quad$ Paucibacilar & 222 & \\
$\quad$ Multibacilar & 417 & \\
\hline
\end{tabular}




\begin{tabular}{lcc}
\hline Grau de incapacidade no diagnóstico & 277 & 43,4 \\
Grau 0 & 212 & 33,2 \\
Grau I & 87 & 13,6 \\
Grau II & 6 & 0,9 \\
Ignorado/branco & 54 & 8,5 \\
Não avaliado & & \\
Modo de entrada & 639 & 86,9 \\
Caso novo & 9 & 1,2 \\
Transferência do mesmo município & 47 & 6,4 \\
Transferência de outro município (leia-se, estado, país) & 23 & 3,1 \\
Recidiva & 17 & 2,4 \\
Outros reingressos & & 31,6 \\
Modo de deteç̧ão & 202 & 55,4 \\
Encaminhamento & 354 & 3,6 \\
Demanda espontânea & 23 & 5,2 \\
Exame de coletividade & 33 & 0,6 \\
Exame de contatos & 4 & 3,6 \\
Ignorado/branco & 23 & \\
Outros modos & & \\
\hline
\end{tabular}

Fonte: Sinan/ Vigilância Epidemiológica - Secretaria de Saúde de Maracanaú/CE. Dados coletados em 6 de fevereiro de 2019

Classificou-se o coeficiente de deteç̧ão de casos novos por 10.000 habitantes como "muito alto" em oito dos dez anos avaliados, sendo também classificado como "alto" em 2013 e "hiperendêmico" em 2018, pois em todos os anos esse coeficiente foi superior a $1 / 10.000$ habitantes (Quadro I).

Já o coeficiente de detecção em menores de 15 anos de idade apresentou flutuações ao longo do período analisado, com valores superiores a 1/10.000 nos anos de 2012, 2014, 2016 e 2018 (Figura II).

Nos anos de 2013 e 2015, a proporção dos casos novos de hanseníase encerrados por cura superou os $90 \%$; e a proporção foi superior a $80 \%$ nos demais anos analisados (Figura III).

Quadro I - Distribuição da detecção de casos novos de hanseníase em Maracanaú de 2009 a 2018. Maracanaú, 2019.

\begin{tabular}{|cccccc|}
\hline Ano & Casos novos & $\begin{array}{c}\text { Coeficiente de } \\
\text { deteção geral por } \\
\mathbf{1 0 0 . 0 0 0} \text { habitantes }\end{array}$ & Classificação* & $\begin{array}{c}\text { Coeficiente de } \\
\text { detecção } \\
\mathbf{0} \text { a } \mathbf{1 4} \text { anos por } \\
\mathbf{1 0 0 . 0 0 0} \text { habitantes }\end{array}$ & Classificação* \\
\hline 2009 & 65 & 3,2 & Muito alto & 0,35 & Médio \\
2010 & 52 & 2,5 & Muito alto & 0,55 & Médio \\
2011 & 71 & 3,3 & Muito alto & 0,37 & Médio \\
2012 & 54 & 2,5 & Muito alto & 1,09 & Alto \\
2013 & 41 & 1,9 & Alto & 0 & Baixo \\
2014 & 58 & 2,6 & Muito alto & 1,06 & Alto \\
2015 & 67 & 3,0 & Muito alto & 0,71 & Médio \\
2016 & 69 & 3,1 & Muito alto & 1,4 & Médio \\
2017 & 67 & 3,0 & Muito alto & 0,35 & Alto \\
\hline 2018 & 95 & 4,2 & Hiperendêmico & 1,79 & - \\
\hline TOTAL & 639 & - & - & - & - \\
\hline
\end{tabular}

Fonte: Sinan/ Vigilância Epidemiológica - SMS Maracanaú/CE. Dados coletados em 6 de fevereiro de 2019

*Hiperendêmico: $\geq 4,0 / 10.000$ hab.; Muito Alto: 4,0 - 2,0/10.000 hab.; Alto: 2,0 - 1,0/10.000 hab.; Médio: 1,0 - 0,2/10.000 hab.; Baixo: $<0,2 / 10.000$ habitantes 


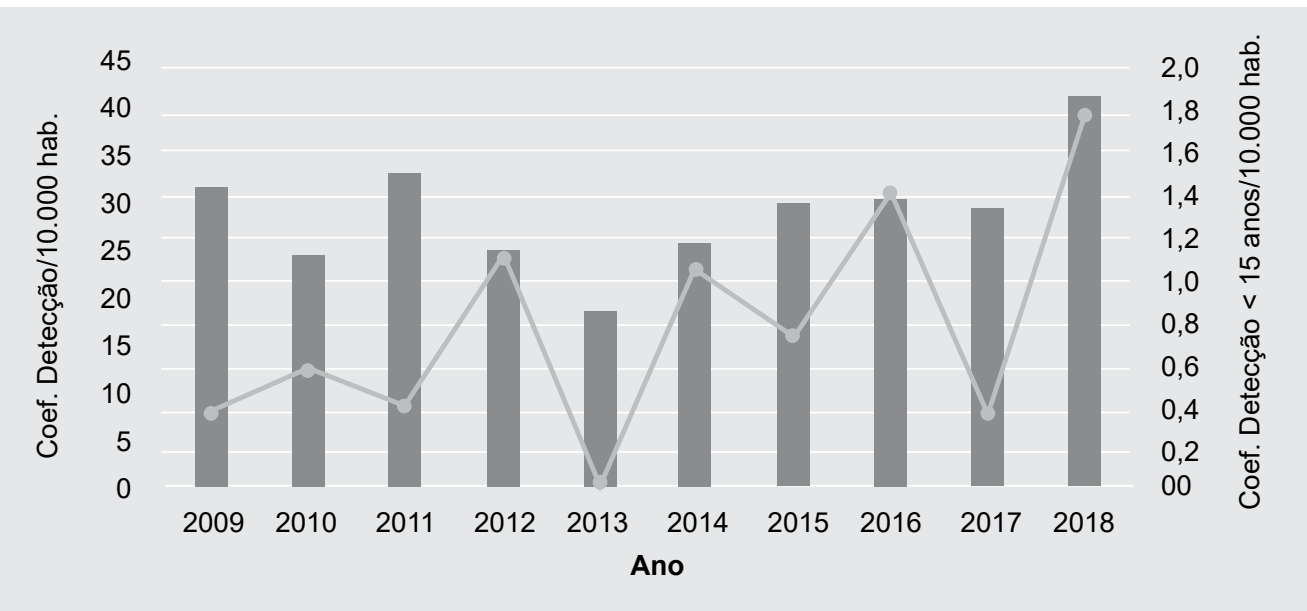

Figura II - Coeficiente de detecção de casos novos por ano em Maracanaú de 2009 a 2018. Maracanaú, 2019.

Fonte: Sinan/ Vigilância Epidemiológica - Secretaria de Saúde de Maracanaú/CE. Dados coletados em 6 de fevereiro de 2019

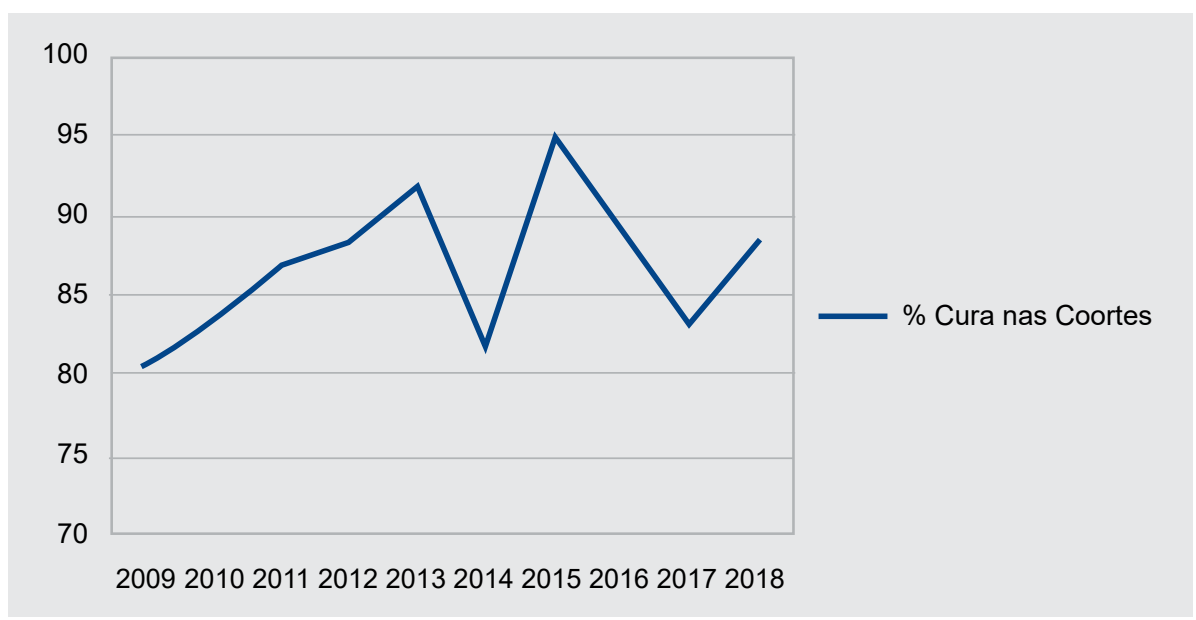

Figura III - Proporção de encerramento por cura dos casos novos de hanseníase no município de Maracanaú de 2009 a 2018. Maracanaú, 2019.

Fonte: Sinan/ Vigilância Epidemiológica - Secretaria de Saúde de Maracanaú/CE. Dados coletados em 6 de fevereiro de 2019

\section{DISCUSSÃO}

Nos últimos anos, houve uma redução do número de casos de hanseníase em nível mundial, entretanto a sua eliminação em alguns países, incluindo o Brasil, ainda é desafiadora. Apesar do empenho da Organização Mundial de Saúde (OMS) e dos governos em erradicar a doença, ela segue como um grande problema de saúde pública(10).

O atual estudo apontou que, no período de 2009 a 2018, o diagnóstico de hanseníase no munícipio de Maracanaú foi mais frequente no sexo masculino. Segundo dados do Ministério da Saúde, no período de 2012 a 2016, observouse que a taxa de detecção por 100 mil habitantes na população masculina foi maior que na população feminina em todas as faixas etárias ${ }^{(4)}$.

Nesse aspecto, devem-se priorizar ações de educação em saúde, vigilância de contatos, qualificação do diagnóstico, prevenção e tratamento de incapacidades, com enfoque diferenciado para homens e mulheres, influenciando os homens para ações de autocuidado e busca pelos serviços de saúde na fase inicial da doença, evitando-se diagnóstico tardio e, consequentemente, incapacidades físicas. A taxa de detecção anual, no período de 2018, foi de 4,2/10.000 mil habitantes, interpretada como hiperendêmico, segundo os parâmetros do Ministério da Saúde ${ }^{(4)}$. Entre a população de zero a 14 anos, o coeficiente de detecção variou, no período de 2015 a 2018, entre médio e alto. Esse parâmetro avalia a força da transmissão recente da endemia e sua tendência.

A série histórica permitiu avaliar que a classificação clínica dimorfa, que se classifica como multibacilar, permanece com uma representação significativa no município, achado semelhante ao encontrado no estado do Ceará e em outros estudos $(5,6,11)$. 
Com a perspectiva voltada às ações de detecção precoce e redução dos casos novos de hanseníase, o Ministério da Saúde orienta a investigação epidemiológica de todos os contatos intradomiciliares, incluindo: avaliação dermatoneurológico de todos os contatos intradomiciliares dos casos novos de hanseníase e orientações acerca dos sinais e sintomas da doença, período de incubação, transmissão e orientações ${ }^{(3,12)}$.

Essa ação estratégica favorece a deteç̧ão ativa para a descoberta de casos e visa ao diagnóstico precoce, contribuindo para a queda da cadeia de transmissão e reduzindo as incapacidades que surgem em decorrência do diagnóstico tardio. Verificou-se que, no Brasil, no período de 2012 a 2016, $77 \%$ dos contatos dos casos novos de hanseníase diagnosticados foram avaliados, e, entre as regiões, o Nordeste obteve percentual mais baixo $(71,8 \%)^{(4,13)}$.

Em relação ao estado do Ceará, no período de 2008 a 2017 foram registrados 76.029 contatos no Sinan, sendo examinados 53.802 (70,8\%). Dessa forma, houve aumento de 7,2\% na proporção de contatos examinados, passando de $65,4 \%$, em 2008 , para $70,1 \%$, em 2017 , no entanto ainda se mantém abaixo do preconizado pelo Ministério da Saúde(5).

Por sua vez, o município de Maracanaú apresentou, em 2018, uma proporção de exames de contato bem acima da média nacional, contudo os dados revelam, também, que o número de casos diagnosticados através de exame de contato (33 casos) representa 5,2\% do total de diagnósticos, sendo inferior ao encontrado em outros municípios ${ }^{(14,15)}$.

Ainda com relação ao modo de entrada, a demanda espontânea, o encaminhamento e outros modos foram predominantes no presente estudo, em detrimento ao exame de contato, sendo semelhante ao encontrado em estudo(16) que reforça que a menor proporção de casos diagnosticados por exame de contato sugere passividade e/ou negligência dos serviços prestados pelos profissionais da Estratégia de Saúde da Família na busca ativa de casos ou, a ocorrência menor nessa população em razões de fatores imunológicos. Entretanto há necessidade de outros estudos para se avaliar essas hipóteses.

Dentre os casos avaliados quanto ao grau de incapacidade física no momento do diagnóstico, uma quantidade significativa de pacientes tem acesso a essa avaliação, considerado como parâmetro bom, entretanto a proporção de grau 2 no diagnóstico se manteve com parâmetro alto para os casos novos de hanseníase, demonstrando que, embora os pacientes tenham acesso ao serviço em nível municipal, o diagnóstico está sendo realizado tardiamente no município analisado, e devido a doença ser potencialmente incapacitante, poderá ocasionar deformidades físicas, podendo, na fase infantil e da adolescência, ter influência na vida escolar, por conta da limitação social, discriminação, baixa autoestima e do estigma ${ }^{(2,17)}$.

Quanto ao indicador cura, verifica-se um parâmetro regular no município em estudo, com proporção de 88,4\%, semelhante aos dados encontrados no estado do Ceará(5). A cura em hanseníase é a condição na qual o paciente, inicialmente infectado pelo Mycobacterium leprae e diagnosticado como um caso de hanseníase, iniciou e concluiu o tratamento para paucibacilar (PB) ou multibacilar (MB) no prazo estimado pela OMS. Entretanto, em alguns casos, especialmente nos multibacilares, embora o paciente tenha concluído a PQT (tratamento poliquimioterápico) e já tenha ocorrido a sua exclusão do registro ativo dos casos de hanseníase, a pessoa atingida pela doença, geralmente, continua recebendo acompanhamento em decorrência das incapacidades físicas adquiridas e/ou pela possibilidade de apresentar reação hansênica ${ }^{(16)}$.

Nesse sentido, espera-se uma estrutura adequada da rede de atenção em saúde local para que ocorra a avaliação sistemática dos pacientes no pós-alta de hanseníase, pois o adequado manejo dessas pessoas determinará, em grande parte, a prevenção de complicações, a redução de custos com reabilitação e a melhoria da qualidade de vida desses pacientes ${ }^{(13)}$.

No Brasil, a prevenção à hanseníase depende prioritariamente das ações da Equipe de Saúde da Família, as quais permitem um redirecionamento das prioridades em saúde, dentre elas, a do controle da hanseníase destacandose: a vigilância epidemiológica; a gestão com foco na descentralização, no planejamento, no monitoramento e na avaliação; a atenção integral; a comunicação e educação; e o desenvolvimento de pesquisas ${ }^{(18)}$.

Dentre as atividades de promoção da saúde voltadas a essa temática, torna-se imprescindível a participação dos profissionais da Estratégia Saúde da Família, especialmente substituindo metodologias tradicionais, para a utilização de recursos que venham a contribuir para uma prática educativa em uma perspectiva não verticalizada, lúdica, inovadora, criativa, interativa e reflexiva, buscando-se promover o empoderamento dos sujeitos quanto à sua saúde, direitos e condições de vida, ultrapassando as ações que visam apenas à distribuição de poliquimioterapia, o encaminhamento dos pacientes a outros cuidados e o preenchimento de sistemas de informação(19,20).

Para que o Brasil consiga eliminar a hanseníase como um problema de saúde pública, é preciso que os serviços de saúde possuam, portanto, profissionais com capacidade de trabalhar em equipe, de interagir com diversas 
pessoas e segmentos sociais, captando e processando informações por meio da comunicação, e aplicando suas habilidades e competências no exercício diário de seu trabalho ${ }^{(21)}$.

Como limitação do presente estudo, destacam-se questões relacionadas ao banco de dados do Sinan, pois esse sistema pode apresentar informações inconsistentes em termos de quantidade e qualidade, como erros de digitação, inconsistências e duplicação de dados. Entretanto, a despeito das limitações, o estudo traz informações clínicas e epidemiológicas importantes acerca dos pacientes com hanseníase e poderá fornecer subsídios às ações de planejamento, diagnóstico, prevenção, controle e redução das incapacidades físicas características do diagnóstico tardio, favorecendo a qualidade de vida dos pacientes acometidos com a doença.

\section{CONCLUSÃO}

O município analisado encontra-se com taxa de detecção anual que indica hiperendemicidade, indicando alta detecção de casos, e ainda apresenta casos registrados em menores de quinze anos, indicando a permanência de fontes de transmissibilidade. As características clínicas e epidemiológicas dos pacientes com hanseníase são do sexo masculino, classificação operacional multibacilar, sendo a forma clínica dimorfa a mais frequente, com predomínio para o grau zero de incapacidade no momento do diagnóstico.

\section{CONFLITOS DE INTERESSE}

As autoras afirmam que não houve conflitos de interesses na execução desta pesquisa.

\section{CONTRIBUIÇÕES}

Carmem Rita Sampaio de Sousa, Mariana Campos da Rocha Feitosa e Kellyn Kessiene de Sousa Cavalcante contribuíram com a elaboração e delineamento do estudo; a aquisição, análise e interpretação dos dados; a redação do manuscrito. Ana Beatriz Ferreira Pinheiro contribuiu com a aquisição, análise e interpretação dos dados.

\section{FONTES DE FINANCIAMENTO}

Coordenação de Aperfeiçoamento de Pessoal do Ensino Superior (CAPES).

\section{REFERENCIAS}

1. Silva MCD, Paz EPA. Educação em saúde no Programa de Controle da Hanseníase: a vivência da equipe multiprofissional. Esc Anna Nery Rev Enferm [Internet]. 2010 [acesso em 2019 Fev 16];14(2):223-9.

Disponível em: http://www.scielo.br/pdf/ean/v14n2/02.pdf

2. Pires CAA, Malcher CMRS, Abreu JMC Jr, Albuquerque TG, Correa IRS, Daxbacher ELR. Hanseníase em menores de 15 anos: a importância do exame de contato. Rev Paul Pediatr [Internet]. 2012 [acesso em 2019 Fev 16];30(2):292-5. Disponível em: http://www.scielo.br/pdf/rpp/v30n2/22.pdf

3. Ministério da Saúde (BR), Secretaria de Vigilância em Saúde, Departamento de Vigilância das Doenças Transmissíveis. Diretrizes para vigilância, atenção e eliminação da hanseníase como problema de saúde pública. Brasília: Ministério da Saúde; 2016.

4. Ministério da Saúde (BR), Secretaria de Vigilância em Saúde. Indicadores epidemiológicos e operacionais de hanseníase, Brasil 2012-2016 [Internet]. Brasília: Ministério da Saúde; 2018 [acesso em 2019 Fev 23]. Disponível em: http://portalarquivos2.saude.gov.br/images/pdf/2018/janeiro/31/2018-004-Hanseniasepublicacao.pdf

5. Secretaria de Saúde do Estado do Ceará, Coordenadoria de Vigilância em Saúde, Núcleo de Vigilância Epidemiológica, Secretaria de Saúde do Estado do Ceará. Boletim Epidemiológico. 2018 [acesso 2019 Fev 23]. Disponível em: https://www.saude.ce.gov.br/wp-content/uploads/sites/9/2018/06/Boletim-2018_ Cear\%C3\%A1-revisado-FINAL.pdf

6. Lana FCF, Carvalho APM, Davi RFL. Perfil epidemiológico da hanseníase na microrregião de Araçuaí e sua relação com ações de controle. Esc Anna Nery Rev Enferm [Internet]. 2011 [acesso em 2019 Mar 
20];15(1):62-7. Disponível em:http://www.scielo.br/scielo.php?script=sci_arttext\&pid=S141481452011000100 009\&lng=pt. http://dx.doi.org/10.1590/S1414-81452011000100009.

7. Ministério da Saúde (BR), Secretaria de Vigilância em Saúde, Departamento de Vigilância Epidemiológica. Portaria $n^{\circ}$ 3.125/2010. Diretrizes para vigilância, atenção e controle da Hanseníase. Brasília: Ministério da Saúde; 2011.

8. Alencar CHM, Barbosa JC, Ramos AN Jr, Alencar MDJF, Pontes RJS, Castro CGJ, et al. Hanseníase no município de Fortaleza, CE, Brasil: aspectos epidemiológicos e operacionais em menores de 15 anos (19952006). Rev Bras Enferm [Internet]. 2008 [acesso em 2019 Fev 16];61(spe):694-700. Disponível em: http:// www.scielo.br/pdf/reben/v61nspe/a07v61esp.pdf

9. Instituto Brasileiro de Geografia e Estatística. Sinopse do censo demográfico [Internet]. 2010 [acesso em 2018 Jan 15]. Disponível em: http:// www.censo2010.ibge.gov.br

10. Ministério da Saúde (BR), Secretaria de Vigilância em Saúde, Departamento de Vigilância em Doenças Transmissíveis. Plano integrado de ações estratégicas de eliminação da hanseníase, filariose, esquistossomose e oncocercose como problema de saúde pública, tracoma como causa de cegueira e controle de geohelmintíases: plano de ação 2011-2015. Brasília: Ministério da Saúde; 2012.

11. Miranzi SDSC, Pereira LHDM, Nunes AA. Perfil epidemiológico da hanseníase em um município brasileiro, no período de 2000 a 2006. Rev Soc Bras Med Trop [Internet]. 2010 [acesso em 2019 Fev 16];43(1):62-7. Disponível em: http://www.scielo.br/pdf/rsbmt/v43n1/a14v43n1.pdf

12. Brasil. Ministério da Saúde. Gabinete do Ministro. Portaria $n^{\circ} 3125$, de 7 de outubro de 2010. Aprova as diretrizes para vigilância, atenção e controle da hanseníase. Diário Oficial da República Federativa do Brasil, Poder Executivo [Internet]. Brasília, DF. 15 Out. 2010 [acesso 2019 Fev 23]; Seção 1, p.55. Disponível em: http://www.anvisa.gov.br/hotsite/talidomida/legis/portaria_n_3125_hanseniase_2010.pdf

13. Hacker MD, Duppre NC, Nery JAC, Sales AM, Sarno EN. Characteristics of leprosy diagnosed through the surveillance of contacts: a comparison with index cases in Rio de Janeiro, 1987-2010. Mem Inst Oswaldo Cruz [Internet]. 2012 [acesso 2019 Fev 16];107(supl 1):49-54. Disponível em: http://www.scielo.br/pdf/mioc/ v107s1/09.pdf

14. Lobo JR, Barreto JCC, Alves LL, Crispim LC, Barreto LA, Duncan LR, et al. Perfil epidemiológico dos pacientes diagnosticados com hanseníase através de exame de contato no município de Campos dos Goytacazes, RJ. Rev Soc Bras Clin Med [Internet]. 2011 [acesso 2019 Fev 16];9(4):283-7. Disponível em: http://files.bvs.br/upload/S/1679- 1010/2011/v9n4/a2187.pdf

15. Garcia DR, Ignotti E, Cortela DCB, Xavier DR, Barelli CSGAP. Análise espacial dos casos de hanseníase, com enfoque à área de risco, em uma unidade básica de saúde no município de Cáceres (MT). Cad Saúde Colet [Internet]. 2013 [acesso 2019 Fev 16];21(2). Disponível em: http://www.scielo.br/scielo.php?pid=S1414462X2013000200011\&script=sci_arttext

16. Brito KKG, Andrade SSDC, Diniz IV, Matos SDDO, Oliveira SHDSO, Oliveira MJGO. Caracterização dos casos de hanseníase diagnosticados através do exame de contato. Rev Enferm UFPE [Internet]. 2016 [acesso $2019 \mathrm{Fev}$ 16];10(2):435-41. Disponível em: https://periodicos.ufpe.br/revistas/revistaenfermagem/ article/download/10974/12309

17. Vieira MA, Lima RAG. Crianças e adolescentes com doença crônica: convivendo com mudanças. Rev LatinoAm Enferm [Internet]. 2002 [acesso 2019 Fev 16];10(4):552-60. Disponível em: http://www.scielo.br/pdf/ rlae/v10n4/13368.pdf.

18. Leal DR, Cazarin G, Bezerra LCA, Albuquerque AC, Felisberto E. Leprosy Control Program: assessment of implementation in districts. Saúde Debate [Internet]. 2017 [cited 2018 Dec 12];41(spe):209-28. doi: http:// dx.doi.org/10.1590/0103-11042017s16

19. Moreira AJ, Naves JM, Fernandes LFRM, Castro SS, Walsh IAP. Ação educativa sobre hanseníase na população usuária das unidades básicas de saúde de Uberaba-MG. Saúde Debate [Internet]. 2014 [cited 2018 Dec 12];38(101):234-43. Available from: http://www.scielo.br/pdf/sdeb/v38n101/0103-1104sdeb-38-101-0234.pdf 
20. Carvalho FPB, Miranda FAN, Simpson CA, Queiroz TA, Isoldi DMR. The context of the care of the nursing professional for people with leprosy in family health strategy. Rev Pesqui (Univ Fed Estado Rio J) [Internet]. 2015 [cited 2018 Dec 12];7:189-99. Available from: http://www.seer.unirio.br/index.php/cuidadofundamental/ article/view/5926

21. Silva RA Sobrinho, Mathias TAF, Gomes EA, Lincoln PB. Avaliação do grau de incapacidade em hanseníase: uma estratégia para sensibilização e capacitação da equipe de enfermagem. Rev Latino-Am Enferm. 2007;15(6):1125-30.

\section{Endereço do primeiro autor:}

Carmem Rita Sampaio de Sousa

Universidade Federal do Ceará - UFC

Rua Papi Junior, $1223 / 5^{\circ}$ andar

Bairro: Rodolfo Teófilo

CEP: 60430-450 - Fortaleza - CE - Brasil

E-mail: carminhaneri83@gmail.com

\section{Endereço para correspondência:}

Kellyn Kessiene de Sousa Cavalcante

Universidade Federal do Ceará - UFC

Rua Papi Junior, 1223/ $5^{\circ}$ andar

Bairro: Rodolfo Teófilo

CEP: 60430-450 - Fortaleza - CE - Brasil

E-mail: kellynveterinaria@hotmail.com

Como citar: Sousa CRS, Feitosa MCR, Pinheiro ABF, Cavalcante KKS. Aspectos epidemiológicos da hanseníase em um município nordestino do Brasil. Rev Bras Promoç Saúde. 2019;32:9469. 